\section{Long-term efficacy and visual acuity following transscleral diode laser photocoagulation in cases of refractory and non-refractory glaucoma}

${ }^{1}$ Eye Department, Eye, Ear \& Mouth Unit, Maidstone Hospital, Maidstone, Kent, UK

${ }^{2}$ Eye Department, Royal Glamorgan Hospital, Ynysmaerdy, Llantrisant, Mid Glamorgan, UK

Correspondence: E Ansari, Eye Department, Eye, Ear and Mouth Unit, Maidstone Hospital, Maidstone and Tunbridge Wells NHS Trust, Hermitage Lane, Maidstone, Kent, ME16 9QQ, UK Tel: +44622 226275; Fax: + 4462272900 .

E-mail: eaansari@aol.com

Received: 3 October 2005 Accepted in revised form: 20 February 2006 Published online: 21 April 2006

Presented at Royal College of Ophthalmologists Conference, Birmingham, 2005

Proprietary interests: Nil

Research funding: Nil
Abstract

Aims Transscleral diode laser cyclophotocoagulation (TSCP) is widely accepted in the treatment of refractory glaucoma especially in eyes with poor vision. However, until recently, there has been a reluctance to extend its use to eyes with good visual acuity (VA). The aims of this study were to evaluate the long-term efficacy and safety of TSCP for a range of glaucoma conditions, with particular emphasis on post-treatment VA in seeing eyes.

Methods A retrospective analysis of case notes of 74 treated eyes was conducted over a period of 4-30 months (mean 12.5 months).

Results Mean (SD) intraocular pressure (IOP) was reduced by $43 \%$ from 40.3 (6.7) to $21.1 \mathrm{mmHg}(5.4)$ at the final index visit. Of all patients, $58 \%$ had a reduction in glaucoma drops and all discontinued oral Acetazolamide; 9\% experienced complications. Mean VA was preserved in the subgroups with good vision, although $3 / 23(13 \%)$ patients with primary open-angle glaucoma (POAG) lost vision due to cataract and glaucoma progression. There were no cases of hypotony (IOP $\leqslant 5 \mathrm{mmHg}$ ) or phthisis and only one eye required re-treatment.

Conclusions TSCP is highly effective and safe for various types of glaucoma, and its use can be extended to eyes with good vision. Eye (2007) 21, 936-940; doi:10.1038/sj.eye.6702345; published online 21 April 2006

Keywords: glaucoma; cyclodiode; laser; visual acuity; intraocular pressure
E Ansari ${ }^{1}$ and J Gandhewar ${ }^{2}$

Introduction

Transscleral cyclodiode laser cyclophotocoagulation (TSCP) plays an important role in the paradigm of glaucoma treatment, especially for uncontrolled or refractory cases with poor visual acuity (VA). ${ }^{1-5}$ Recently it has gained attention as a modality for treatment of glaucoma cases with good vision, and even as a primary surgical treatment in some situations, ${ }^{5-6}$ thereby establishing a broader role for TSCP. We report the long-term efficacy and safety data in a group of glaucoma patients, with particular emphasis on the long-term preservation of VA in those with ambulatory vision (6/36 or better) pretreatment.

\section{Materials and methods}

A single centre, retrospective case note study was conducted for all patients treated with TSCP by a single practitioner (EAA) at the Royal Glamorgan Hospital from March 2002 until September 2004. Data collected included age, gender, diagnosis, intraocular pressure (IOP) pretreatment, immediately after treatment and at the final index visit. The follow-up period, topical and oral anti-glaucoma medication, complications and the total number of laser treatments were also recorded. Treatment success was defined as IOP reduction of greater than $30 \%$, with or without topical anti-glaucoma medications. Data collection and analysis was done with the assistance of the Audit Department, Royal Glamorgan Hospital, and Glamorgan University. 
The indications for treatment were (1) inadequate control of IOP despite maximum tolerated medical therapy, (2) allergy to antiglaucoma medication or inability to tolerate medication, (3) patient unwilling to have drainage surgery, (4) and painful, blind eye.

The treatment protocol was established following an internal audit, which established the laser settings used in this study to be safe. TSCP was performed on a day case basis using the OcuLight SLx semiconductor diode $810 \mathrm{~nm}$ laser and contact G probe (IRIDEX Corp., Mountain View, CA, USA). Informed consent was taken after the procedure was explained fully in each case. Under peribulbar local anaesthesia, laser energy was delivered through a $600 \mu \mathrm{m}$ diameter $\mathrm{G}$ probe, as described in the previous literature. ${ }^{3,4}$ In repeat cases, or those with abnormal anterior segment morphology, transillumination was performed before laser delivery. The applications were made over $360^{\circ}$, except at 3 and $9 \mathrm{O}^{\prime}$ clock to spare the anterior ciliary vessels and nerves. The default energy level was set at $2 \mathrm{~W}$ and $2 \mathrm{~s}$. Immediately postoperatively, a subconjunctival injection of Mydricaine 2 and Betnesol $4 \mathrm{mg} / \mathrm{ml}$ was given.

Postoperatively, every patient was treated with topical Dexamethasone $0.1 \%$ two hourly along with previous antiglaucoma medications. The Dexamethasone drops were continued and reduced in frequency over a period of 2 months. The anti-glaucoma drugs were reduced in frequency or discontinued at the discretion of the clinician during the follow-up period. The postoperative visits were at 1 week, 1 month, 3 months, 6 months, and every 6 months thereafter up to a maximum of 30 months. At every visit VA, IOP (measured by applanation tonometry), and any complications were noted.

Statistical significance in difference between pre and postoperative IOP was tested using the paired $t$-test, the data being normally distributed, with a $P$-value of $<0.05$ being taken as significant.

A $30 \%$ reduction in IOP with or without medical treatment was regarded as successful.

\section{Results}

These are presented for the entire group and then for the group with good ambulatory vision pre-laser. Cases were identified retrospectively via the operating theatre logbook.

\section{The entire group results}

A total of 74 eyes were treated with a mean (SD) followup of 12.5 (3.6) months (range 4-30 months). Mean age (SD) was 76 (8.7) years (range 40-92 years). There were 38 males and 36 females. All patients were Caucasian.

Neovascular glaucoma (NVG) accounted for $54 \%$ of patients, primary open-angle glaucoma (POAG) 31\%, and others were 15\% (chronic angle closure (CACG), secondary glaucoma). The mean (SD) laser power was 2069 (101) $\mathrm{mW}$ (range 1750-2200 mW). The mean (SD) laser burns was 30 (4.5) (range 15-40). Duration of laser burns was $2000 \mathrm{~ms}$ in all cases. Only one patient (rubeotic glaucoma) had more than one session of treatment.

Efficacy data for the entire group are shown in Table 1. Successful IOP lowering was defined as a $30 \%$ reduction with or without drops. The IOP lowering effect was statistically significant $(P=0.005$, paired $t$-test).In all, $82 \%$ of the patients had more than a $30 \%$ reduction in IOP (Figure 1 and Table 2).

Figure 2 is a Kaplan-Meier plot showing the cumulative probabilities ( $y$-axis) with time ( $x$-axis) that the IOP will be maintained at a $30 \%$ reduction following a course of cyclodiode treatment.

Of all the patients, $58 \%$ had a reduction in the number of antiglaucoma medications. Oral Acetazolamide was stopped after laser treatment in all cases. It was not restarted in any patients during the course of the study.

The overall complication rate of TSCP was $9 \%$. Complications included hyphema $(n=3)$, chronic iritis (>2 months post-treatment) $(n=3)$, and corneal oedema $(n=1)$. None of the patients had vitreous haemorrhage, phthisis bulbi, atonic pupil or prolonged hypotony $(\leqslant 5 \mathrm{mmHg})$. All three cases of hyphema resolved

Table 1 IOP lowering effect of cyclodiode laser for each diagnostic category

\begin{tabular}{lcccccccc}
\hline $\begin{array}{l}\text { Diagnostic } \\
\text { category }\end{array}$ & $\begin{array}{c}\text { No. of eyes } \\
\text { \% total) }\end{array}$ & $\begin{array}{c}\text { Mean }(S D) \\
\text { age of } \\
\text { patients }\end{array}$ & $\begin{array}{c}\text { Mean no. of } \\
\text { treatments }\end{array}$ & $\begin{array}{c}\text { Mean pre- } \\
\text { Laser IOP }\end{array}$ & $\begin{array}{c}\text { Mean post- } \\
\text { Laser IOP }\end{array}$ & $\begin{array}{c}\text { Mean } \\
\text { percentage of } \\
\text { change in IOP }\end{array}$ & $\begin{array}{c}\text { Mean VA } \\
\text { pre }\end{array}$ & $\begin{array}{c}\text { Mean VA } \\
\text { post }\end{array}$ \\
\hline All eyes & $74(100)$ & $75.7(8.7)$ & 1.01 & 40.3 & 21.1 & 45.1 & \\
Neovascular & $37(50)$ & $75.1(8.3)$ & 1.02 & 48.2 & 25.3 & 47.0 & $6 / 22$ & $6 / 19$ \\
POAG & $23(31)$ & $79.2(10.8)$ & 1.0 & 28.6 & 16.1 & 39.0 & $6 / 26$ \\
CACG & $6(8)$ & $66.7(7.1)$ & 1.0 & 35.0 & 23.0 & 29.9 & $6 / 29$ \\
\hline
\end{tabular}

Efficacy data for the entire group and for subgroups.

$\mathrm{POAG}=$ primary open-angle glaucoma; $\mathrm{CACG}=$ chronic angle closure glaucoma . 


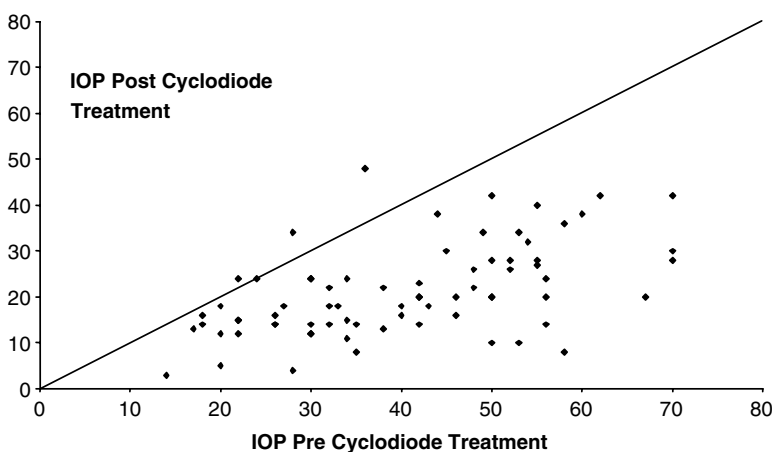

Figure 1 Scatter plot showing the change in IOP following TSCP. All points below the diagonal line represent eyes that responded with a fall in IOP.

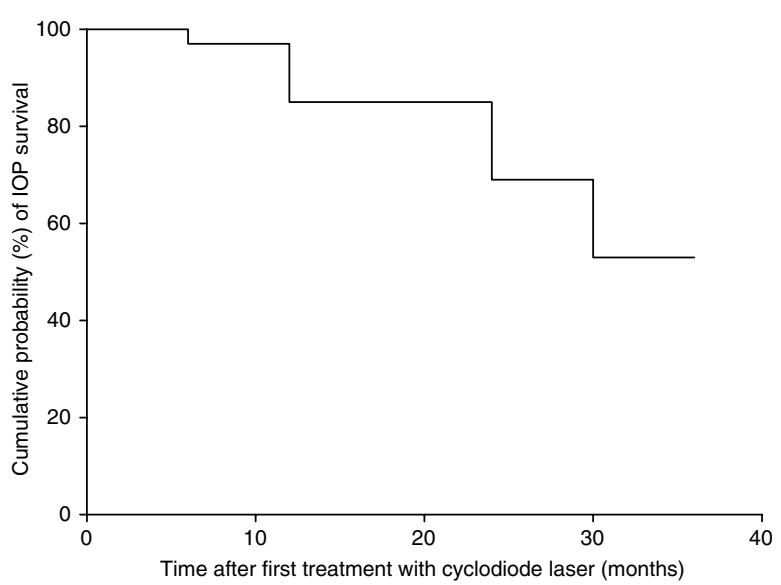

Figure 2 Kaplan-Meier plot showing the cumulative probabilities ( $y$-axis) with time ( $x$-axis) that the IOP will be maintained at a $30 \%$ reduction following a course of cyclodiode treatment.

spontaneously. Persistent iritis was seen in three patients with CACG. All of these had an existing patent peripheral iridotomy.

\section{Results for the POAG group with good pre-laser vision}

A total of 23 eyes with POAG were treated in this group. Table 1 summarises the efficacy data for this group. The mean (SD) follow-up period was 12.1 (4.4) months (range 6-27 months). There were 13 males and 10 females. The mean (SD) age was 79.2 (10.8) years.

The mean (SD) laser power was 1997 (93) $\mathrm{mW}$ (range 1750-2100 mW). The mean (SD) laser burns was 30 (4.5) (range 15-40). Duration of laser burns was $2000 \mathrm{~ms}$ in all cases. All patients had one treatment session.

The mean pre-laser VA was 6/22 (range 6/36-6/6). The mean pre-laser VA did not deteriorate following treatment. Although the mean VA did not decrease after treatment, the final VA was worse in three cases (13\%). In
Table 2 Success of TSCP: data for the entire group and subgroups

\begin{tabular}{lcc}
\hline Diagnosis & No. of eyes (\% total) & Success rate (\%) \\
\hline All eyes & $74(100)$ & 82.4 \\
Neovascular & $37(50)$ & 75.7 \\
POAG & $23(31)$ & 91.3 \\
CACG & $6(8)$ & 83.3 \\
\hline
\end{tabular}

two cases there was a worsening of lens opacities and in one case there was chronic anterior uveitis and a progression of optic neuropathy (vertical optic disc excavation from 0.8 to 0.95 by stereoscopic optic disc photography).

Of all the patients, $52 \%$ had a reduction in the number of antiglaucoma medications. Oral Acetazolamide was stopped post laser treatment in all cases.

In all, $13 \%$ of cases $(n=3)$ had complications, of which there was hyphema $(n=2)$, and anterior uveitis $(n=1)$.

In the smaller group $(n=8)$ of patients with CACG and good vision, mean VA did not deteriorate significantly after TSCP $(P=0.5)$.

We compared the results between rubeotic glaucoma and POAG. In all, 17\% of POAG patients showed improvement of VA. In $75.7 \%$ of rubeotic glaucoma, there was $>30 \%$ reduction in IOP as compared to $91.3 \%$ in POAG (Table 2).

\section{Discussion}

The $810 \mathrm{~nm}$ diode laser has proven to be efficacious in IOP reduction in glaucoma and has other advantages such as portability and adaptability in terms of mode of energy delivery. ${ }^{3}$ The $810 \mathrm{~nm}$ diode wavelength is twice as efficient for TSCP than the Nd:YAG wavelength at $1064 \mathrm{~nm}$, and is absorbed better by melanin. ${ }^{2}$ It is accepted as a safe treatment modality in complex and refractory glaucomas, with minimal pain and inflammation. $^{1-3,7}$ TSCP is quick, easy to learn and does not require a sterile operating room. ${ }^{5}$

Ideally, any treatment should reduce IOP to a satisfactory level while preserving pretreatment visual function. The reason for cyclodestructive procedures not being widely used as a primary procedure in seeing eyes was because of complications reported in very early studies. ${ }^{8,9}$ This study confirms the findings of previous studies, ${ }^{1-9}$ which demonstrated safety and efficacy to reduce IOP in complex cases. Furthermore, it confirms efficacy and preservation of visual function in patients with good VA pre-laser. Treatment also helped to reduce, although not eliminate, the total number of drops used after laser treatment, and enabled cessation of treatment with oral Acetazolamide. This is a major quality of life 
issue for patients as oral Acetazolamide is associated with so many undesirable side effects.

According to the definition of success used in this study, $82 \%$ of eyes had a successful reduction of IOP at final follow-up. In all cases, a satisfactory IOP was achieved with topical treatment, although the total number of medications used was reduced after TSCP. Oral Acetazolamide was discontinued for the duration of the study. Mean IOP was significantly reduced by $45.1 \%$ (40.3-21.1 $\mathrm{mmHg})(P=0.005$, paired $t$-test). This compares favourably with other studies, ${ }^{1-9}$ although direct comparisons are difficult because of different study populations and treatment protocols. ${ }^{4}$ Other cyclodestructive procedures (cyclocryotherapy, Nd:YAG cycloablation) are associated with reduction of IOP ranging between 43 and 51\% with 12-30 months follow-up, ${ }^{10,11}$ although there are more side-effects and vision loss with those methods. ${ }^{12}$

It is encouraging that in the subgroups with good pre-laser vision, the mean VA post-TSCP was no worse than the pre-laser level, indeed slightly better in the case of POAG patients. Individually, 3 of the 23 cases in this subgroup did experience a reduction of VA post TSCP.

Two cases were due to cataract, a reversible cause of VA reduction, and one case was due to worsening of glaucomatous optic neuropathy (GON). These complications are not limited to TSCP as incisional drainage surgery is also associated with these problems. ${ }^{13}$ The CACG subgroup of patients also benefited from TSCP, with preservation of vision. In a recent study in South-East Asian patients, TSCP as primary surgical treatment was safe and effective with a $38.5 \%$ risk of visual deterioration (5 out of 13 patients). As in our study, the visual decline was attributed to cataract and progression of GON. ${ }^{6}$

Recent studies have been conducted on the earlier use of TSCP including those cases with good VA. A retrospective study of 21 eyes with VA 20/80 or better demonstrated that in most cases VA was preserved post TSCP. ${ }^{14}$ In a study in Ghana, TSCP was used as primary surgical treatment. There was a reduction of VA in $23 \%$ of cases, but also a similar reduction in fellow eyes treated with medications alone. ${ }^{5}$ Other studies such as the Advanced Glaucoma Intervention Study (AGIS) ${ }^{15}$ demonstrated a reduction of VA over time following conventional procedures such as incisional drainage surgery and argon laser trabeculoplasty (ALT). Therefore, the decrease in vision following treatment is not specific to cyclodestruction.

We were pleased with the clinical results of the laser treatment and the low complication rate. Our retreatment rate and complications were lower than previous studies. ${ }^{2,3,7,14}$ This is probably because the group of patients studied were all Caucasians with fewer complex problems and co-morbidity, and also because of a larger subgroup (23/74) who had TSCP as the primary surgical treatment. Results and conclusions from previous studies helped us to adapt our treatment protocols and postoperative management to reduce long-term problems. Close postoperative supervision in a dedicated Glaucoma clinic setting was beneficial. Complications such as atonic pupil and hypotony were avoided. We believe that avoiding treatment to the 3 and 9 O'clock positions is pupil-sparing as the anterior ciliary neurovascular structures are not damaged. One patient required retreatment and we ensured that the power settings at re-treatment were reduced in order to avoid hypotony. ${ }^{16}$

We acknowledge the limitations of this study. It was retrospective and non-comparative. Quantitative VA data would be improved using Logmar acuity testing. Grading and follow-up of ocular co-morbidity such as cataract would enable a better system of documenting VA loss due to other ocular conditions. A prospective comparison with medications, other laser treatments such as ALT and incisional drainage surgery would be ideal. However, the study does have a reasonable number of cases and a follow-up period of up to 30 months.

We believe that the results of this study are encouraging and support the view that TSCP can be used safely and successfully in seeing eyes, therefore extending the role of TSCP in glaucoma management. ${ }^{17}$

\section{References}

1 Hawkins TA, Stewart WC. One-year results of semiconductor transscleral cyclophotocoagulation in patients with glaucoma. Arch Ophthalmol 1993; 111: 488-491.

2 Kosoko O, Gaasterland DE, Pollack IP, Enger CL. Long term outcome of initial ciliary ablation with contact diode laser transscleral cyclophotocoagulation for severe glaucoma. Ophthalmology 1996; 103: 1294-1302.

3 Bloom PA, Tsai JC, Sharma K, Miller MH, Rice NS, Hitchings RA et al. Cyclodiode: transscleral diode laser cyclophotocoagulation in the treatment of advanced refractory glaucoma. Ophthalmology 1997; 104: 1508-1519.

4 Spencer AF, Vernon SA. 'Cyclodiode': results of a standard protocol. Br J Ophthalmol 1999; 83: 311-316.

5 Egbert PR, Fiyadoyor S, Budenz DL, Dadzie P, Byrd S. Diode laser transscleral cyclophotocoagulation as a primary surgical treatment for primary open angle glaucoma. Arch Ophthalmol 2001; 119: 345-350.

6 Lai JSM, Tham CCY, Chan JCH, Lam D. Diode laser transscleral cyclophotocoagulation as primary surgical treatment for medically uncontrolled chronic angle closure glaucoma. J Glaucoma 2005; 14(2): 114-119.

7 Brancato R, Carassa RG, Bettin P. Contact transscleral cyclophotocoagulation with diode laser in refractory glaucoma. Eur J Ophthalmol 1995; 5: 32-39. 
8 Benson MT, Nelson ME. Cyclocryotherapy: a review of cases over a 10 year period. Br J Ophthalmol 1990; 74: 103-105.

9 Caprioli J, Strang SL, Spaeth GL. Cyclocryotherapy in the treatment of advanced glaucoma. Ophthalmology 1985; 92: 947-954.

10 Schuman JS, Bellows AR, Shingleton BJ. Contact transscleral $\mathrm{Nd}$ :YAG laser cyclophotocoagulation. Midterm results. Opphthalmology 1992; 99: 1089-1095.

11 Dickens CJ, Nguyen N, Mora JS, Iwach AG, Gaffney MM, Wong PC et al. Long-term results of noncontact transscleral neodymium:YAG cyclophotocoagulation. Ophthalmology 1995; 102: 1777-1781.

12 Youn J, Cox TA, Herndon LW, Rand Allingham R, Shields MB. A clinical comparison of transscleral cyclophotocoagulation with neodymium:YAG and semiconductor diode lasers. Am J Ophthalmol 1998; 12 640-647.
13 Migdal C, Gregory W, Hitchings RA. Long-term functional outcome after early surgery compared with laser and medicine in open-angle glaucoma. Ophthalmology 1994; 101: 1651-1657.

14 Wilensky J, Kammer J. Long-term visual outcome of transscleral laser cyclotherapy in eyes with ambulatory vision. Ophthalmology 2004; 111: 1389-1392.

15 The AGIS Investigators. The advanced glaucoma intervention study (AGIS): 4. Comparison of treatment outcomes within race. Ophthalmology 1998; 105: 1146-1164.

16 Murphy CC, Burnett CAM, Spry PGD, Broadway DC Diamond JP. A two centre study of the dose-response relation for transscleral diode laser cyclophotocoagulation in refractory glaucoma. $\mathrm{Br}$ J Ophthalmol 2003; 87: 1252-1257.

17 Wilson MR. A new role for diode laser transscleral cyclophotocoagulation? Arch Ophthalmol 2001; 119: 424-425. 